\title{
Promising Metamaterials for Optoelectronics based on Polymer Nanocomposites
}

\author{
Ushakov NM*1,2 and Kosobudskii ID ${ }^{1,3}$ \\ ${ }^{1}$ VA Kotel'nikov Institute of Radio Eng \& Electr of RAS, Russia \\ ${ }^{2}$ National Research University, Russia \\ ${ }^{3} Y u$ Gagarin Technical State University, Russia
}

Submitted: June 04, 2020; Published: July 02, 2020

*Corresponding author: Ushakov NM, VA Kotel'nikov Institute of Radio Eng \& Electr of RAS, National Research University, Saratov, Russia

Keywords: Promising metamaterials; Optoelectronics; Nanocomposites; Polymer; electronic equipment; Nanotechnology; Nanoparticles; Materials

\section{Mini Review}

Researchers and developers of electronic equipment devote considerable attention to the search for new physical principles for the organization of artificial electronic media. Recently, new approaches to the structural organization of such media have become possible thanks to the development of nanotechnology. One of the important directions in the development of nanotechnology is the creation and study of nanocomposites based on metal nanoparticles and their compounds stabilized in the volume of a polymer dielectric matrix. The use, as matrices of stabilizers of nanoparticles, polymers, led to the development of a new scientific field - plastic electronics. The properties of metal-containing nanocomposite materials critically depend on the volume concentration of nanoparticles. A change in the concentration of nanoparticles relative to the polymer matrix of the stabilizer significantly affects the basic parameters of the nanomaterial - electrical conductivity, complex dielectric constant, plasma frequency, etc. By varying the composition of the resulting nanocomposite, by changing the type of dielectric matrix, size, composition and concentration of nanoparticles in it, one can purposefully control the basic physical properties of the nanomaterial - electrical, optical, galvanomagnetic, etc.

Nanomaterials consisting of $\mathrm{Fe} / \mathrm{Fe}_{2} \mathrm{O}_{3}, \mathrm{Cu} / \mathrm{Cu}_{2} \mathrm{O}(\mathrm{CuO}), \mathrm{ZnO}$, CdS nanoparticles stabilized in low density polyethylene (LDPE) were selected as polymer nanocomposites as objects of study. The studies were carried out both on powder samples and on materials in the form of a film with a thickness of 15-50 microns and plates with a thickness of 400-1000 microns. The synthesis of metal-containing nanocomposites was carried out by the method of high-speed thermal decomposition of solutions of metal-containing compounds. Iron pentacarbonyl was used as starting compounds to obtain iron-containing nanoparticles, and corresponding acetates were used for copper and zinc-containing ones. Obtaining nanomaterials was carried out in a solution-melt of polyethylene in purified mineral oil in an argon atmosphere at the decomposition temperature of the above precursors. The gas feed rate was controlled in such a way as to ensure fast and complete removal of ligands and solvent from the reactor. The calculated amount of the solution containing the precursor was introduced into the melt of high-pressure polyethylene with vigorous stirring. After washing the oil with hexane in a Soxhlett apparatus, the samples were dried in vacuum and stored until studies in air began. The resulting materials were powders whose color depended on the concentration and nature of the nanoparticles. The materials necessary for the study of the mold were made from the materials obtained by hot pressing.

The choice of samples for the study was not random but was based on their possible further practical use. The samples studied in the work on the expected properties can be divided into three groups:

a) Electrically conductive (copper-containing nanocomposites).

b) Magnetic (iron-containing nanocomposites).

c) Semiconductor based on transition metal oxides. 
The basic electrophysical and optical properties of samples with different concentrations of metal-containing nanoparticles inside the polymer matrix were investigated, measurements were carried out both at room temperature and heating to $120^{\circ} \mathrm{C}$. Using transmission electron microscopy, it was found that metal atoms (Fe, $\mathrm{Cu}, \mathrm{Zn}$ ) form nanoparticles in the LDPE matrices, the sizes of which depend on the concentration and nature of the metals. The sizes of iron, copper, and zinc containing nanoparticles in the studied samples were: $1-10 \mathrm{~nm}, 10-25 \mathrm{~nm}$ and $2-10 \mathrm{~nm}$, respectively. The results of measuring the passage of direct current through copper-containing nanocomposites at different temperatures showed that contact phenomena are clearly manifested at low temperatures. With a further increase in temperature, the resistance of the sample decreases, and the current throughput increases, which reflects the semiconductor nature of the studied nanomaterials. The study of the temperature dependences of the passage of current through copper-containing samples of nanocomposites revealed an interesting feature, which consists in observing the current hysteresis during heating and cooling of the samples. The conductivity, dielectric constant, and dielectric loss tangent of copper-containing nanocomposites were studied, and the concentration dependence of these properties was determined for these materials. With an increase in the concentration of nanoparticles in the polymer matrix, the dielectric constant increases by six times, losses at a frequency of $1 \mathrm{MHz}$ increase by a factor of three, and conductivity increases by almost 1.5 times. A specific feature of the electrophysical properties of nanocomposites based on copper-containing nanoparticles was found, consisting in a decrease in electrical conductivity in the range of $10-20 \mathrm{wt} . \%$.

From the spectra of optical reflection and transmission of nanocomposites at room temperature, dispersion dependences of the refractive index and absorption coefficient are obtained. It has been established that for nanomaterials with particle sizes of not more than $4 \mathrm{~nm}$, local maxima of the absorption coefficient are observed associated with optical resonant transitions between minibands in the energy spectrum. With an increase in the concentration of nanoparticles in the matrix, the size of the nanoparticles increases, and the maximum luminescence intensity shifts to a longer wavelength region of the spectrum, which is consistent with independent measurements of the shift of the fundamental absorption edge in such materials by other optical methods such as absorption spectroscopy. The results of measurements of the electrophysical and optical properties of bulk polymer nanocomposites show the possibility of effective control of the physical properties of such artificial media at a filling concentration significantly lower than critical $\left(x<x_{c}=50 \%\right)$.
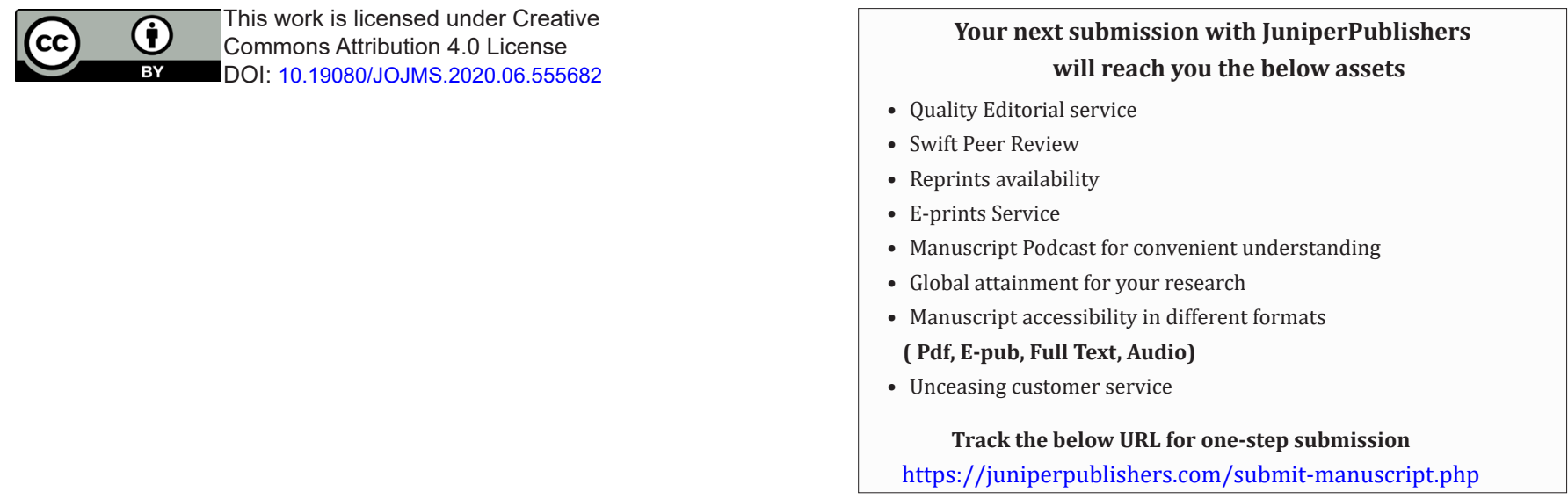